Revista de
Economild
Contemporâned

\title{
OS IMPACTOS DA POLÍTICA MONETÁRIA NA TAXA DE CÂMBIO NO BRASIL: IDENTIFICAÇÃO VIA HETEROCEDASTICIDADE
}

\author{
Sílvia Verônica Vilarinho Couto ${ }^{a}$ \\ Roberto Meurer ${ }^{b}$ \\ ${ }^{a}$ Estudante do doutorado em Economia pelo Programa de Pós-Graduação \\ em Economia da Universidade Federal de Santa Catarina. \\ bProfessor titular do Departamento de Economia e Relações \\ Internacionais daUniversidade Federal de Santa Catarina.
}

Artigo recebido em 05/08/2016 e aprovado em 03/08/2017.

RESUMO: O presente trabalho busca analisar os efeitos da política monetária brasileira sobre a taxa de câmbio, no período de 2004-2016, considerando o problema de endogeneidade da política monetária. Para tratar do problema da endogeneidade da política monetária é empregado o método de identificação via heterocedasticidade. A principal vantagem deste método é que ele se baseia no comportamento estatístico das séries, não sendo necessário realizar suposições arbitrárias de identificação. Os resultados mostram que há uma relação positiva entre a taxa de juros e a taxa de câmbio no Brasil.

PALAVRAS-CHAVE: política monetária; taxa de juros; taxa de câmbio.

CLASSIFICAÇÃO JEL: E43; E52; F31.

Correspondência para: Sílvia Verônica Vilarinho Couto

Contato: veronicavilarinho@gmail.com 


\title{
THE IMPACT OF MONETARY POLICY ON THE EXCHANGE RATE IN BRAZIL: IDENTIFICATION THROUGH HETEROCEDASTICITY
}

\begin{abstract}
This study aims to analyze the effects of Brazilian monetary policy on the exchange rate in from 2004 to 2016 taking into account the endogeneity problem of monetary policy. To address the issue of endogeneity of monetary policy the method of identification through heteroscedasticity is used. The main advantage of this method is that it is based on the statistical behavior of the series, it is not necessary to make arbitrary assumptions of identification. The results show that there is a positive relationship between the interest rate and the exchange rate in Brazil.
\end{abstract}

KEYWORDS: monetary policy; interest rate; exchange rate. 


\section{INTRODUÇÃO}

Embora a relação entre política monetária e a taxa de câmbio seja um tema amplamente debatido na literatura econômica, não há um consenso sobre qual é o sinal dessa relação. A simultaneidade que existe entre essas variáveis torna a relação ainda mais complexa e difícil de ser analisada.

Os modelos mais populares sobre a determinação da taxa de câmbio derivam da combinação entre expectativas racionais, paridade descoberta da taxa de juros e paridade do poder de compra, como Dornbusch (1976), Kouri (1976), Frenkel e Mussa (1984), entre outros. Nesse contexto, um aumento da taxa de juros torna o investimento no país mais atrativo e a taxa de câmbio à vista se aprecia mantendo constante a expectativa futura da taxa de câmbio.

Graças a essa relação, a taxa de câmbio é considerada um dos principais canais de transmissão de política monetária. Uma contração monetária (elevação na taxa de juros) atrai capital externo levando a uma apreciação na moeda doméstica, reduzindo a demanda pela produção doméstica e amenizando pressões inflacionárias. O inverso aconteceria em caso de redução da taxa de juros.

Baseado na visão de que um aumento na taxa de juros causa uma apreciação da moeda local, diferentes países adotaram a política de juros altos em períodos de crises cambiais a fim de proteger a sua moeda. Isso foi muito evidente durante a crise asiática na década de 1990. Porém, nem todos os países que adotaram essa postura conseguiram evitar grandes depreciações da moeda, suscitando novamente o debate sobre a relação entre taxa de juros e taxa de câmbio.

Diversos autores se dedicaram a estudar os efeitos da taxa de juros na taxa de câmbio, especificamente em períodos de crise, como Goldfajn e Gupta (1999), Furman e Stiglitz (1998), Gould e Kamin (2000), Caporale, Cipollini e Demetriades (2005), entre outros. No entanto, as evidências são mistas, um aumento na taxa de juros pode causar tanto uma apreciação na moeda quanto uma depreciação, o que gerou um grande debate sobre a eficácia das taxas de juros em períodos de crise cambial.

As evidências são mistas não só para os períodos de crise, criando assim uma divisão na literatura sobre taxa de câmbio e taxa de juros. A chamada visão tradicional postula que um aumento na taxa de juros leva a uma apreciação da taxa de câmbio, como já citado. Esses modelos se baseiam em fortes suposições, como a validade da paridade do poder de compra e expectativas racionais.

Do outro lado temos a chamada ótica revisionista, ou não tradicional, que postula uma relação positiva entre taxa de juros e taxa de câmbio, ou seja, um aumento na taxa de juros causa uma depreciação na taxa de câmbio. Isso pode ocorrer por fatores diversos, como risco de default, instabilidade no mercado de crédito, desequilíbrios 
do balanço de pagamentos, ambiente inflacionário e outros fatores estruturais de uma economia. O maior risco dos ativos externos leva à depreciação da moeda local e à necessidade de elevação dos juros para controle da inflação, ou seja, esse canal gera uma relação positiva entre depreciação da moeda e juros.

Essas duas óticas esbarram no problema de endogeneidade da política monetária. De acordo com Zettelmeyer (2000), a forma de tratar esse problema pode ser a origem das evidências mistas. Para tratar do problema de identificação da política monetária existem desde modelos de estudos de eventos até modelos de vetores autorregressivos com mudança de regime. Em alguns casos a mesma abordagem produz resultados diferentes, simplesmente pela forma como o problema de endogeneidade é tratado.

O objetivo deste trabalho é verificar os impactos da política monetária sobre a taxa de câmbio utilizando o método de identificação via heterocedasticidade desenvolvido por Rigobon (2003) e Rigobon e Sack (2004). Esse método é empregado por Gonçalves e Guimarães (2011) para o caso da economia brasileira entre os anos 2000 e 2006, cujos resultados mostram uma relação positiva entre a variação da taxa de juros e a variação da taxa de câmbio. No entanto, além de considerar um período mais longo, este trabalho realiza algumas extensões importantes na abordagem de Gonçalves e Guimarães (2011), como a inclusão de variáveis que captam risco, que permitem verificar hipóteses como a dominância fiscal, e a estimação pelo método generalizado dos momentos, além do método de variáveis instrumentais.

O presente trabalho está dividido em cinco seções, incluindo esta introdução. A segunda apresenta os aspectos teóricos da relação entre a política monetária e a taxa de câmbio. A terceira seção apresenta algumas evidências no caso brasileiro. A quarta seção apresenta a metodologia e os resultados estimados. Por fim, são apresentadas algumas considerações finais.

\section{OS EFEITOS DA POLÍTICA MONETÁRIA NA TAXA DE CÂMBIO: ASPECTOS TEÓRICOS}

De acordo com Zettelmeyer (2000), há três motivos para analisar os efeitos da política monetária sobre a taxa de câmbio: avaliar a dinâmica da taxa de câmbio; compreender os efeitos da política monetária, visto que a taxa de câmbio é um dos seus principais mecanismos de transmissão; e verificar se a política monetária é capaz de estabilizar a moeda em períodos de crise e de ataques especulativos.

Considerando o papel de mecanismo de transmissão da política monetária, principalmente em um regime de metas de inflação, como é o caso brasileiro, a taxa de câmbio desempenha um papel fundamental na economia. De acordo com Mendonça (2001), uma política monetária contracionista, ou seja, um aumento na taxa básica de 
juros induz a entrada de capital na economia apreciando a moeda doméstica. Assim, o nível de exportações líquidas se reduz e leva a uma queda na demanda agregada, aliviando a pressão inflacionária. A taxa de câmbio tem um efeito direto e indireto na demanda agregada. $\mathrm{O}$ efeito direto se dá por meio do preço dos bens comercializáveis, enquanto o efeito indireto seria resultado da substituição de bens domésticos por estrangeiros e da redução do preço de insumos importados.

Porém, mesmo sendo amplamente discutido na literatura econômica, ainda há dúvidas sobre como a taxa de câmbio é, de fato, afetada pela política monetária. De modo geral é possível identificar duas vertentes sobre a relação entre política monetária e taxa de câmbio, a convencional ou tradicional e a não convencional, não tradicional ou revisionista, muitas vezes também chamada de relação perversa ou reversa entre taxa de câmbio e política monetária.

Segundo Backus e Driffill (1985), a versão convencional descreve a relação em que um aperto monetário leva à apreciação da taxa câmbio, sendo esta a visão utilizada como base pelo Fundo Monetário Internacional (FMI). Um aumento da taxa de juros torna o investimento em títulos no país mais atrativo, e a taxa de câmbio à vista se aprecia mantendo constante a expectativa futura da taxa de câmbio. Esse resultado deriva da combinação entre expectativas racionais, paridade descoberta da taxa de juros e paridade do poder de compra, como descrito por Dornbusch (1976), Kouri (1976), Frenkel e Mussa (1984), entre outros. O mecanismo de transmissão da taxa de câmbio descrito acima também é baseado nessa visão.

Já a visão não convencional descreve a relação que ocorre quando um aumento na taxa de juros doméstica leva a uma depreciação da taxa de câmbio. Furman e Stiglitz (1998) apresentam alguns motivos que justificam a existência dessa relação reversa. Primeiro, deve-se considerar que a política monetária é capaz de afetar o valor esperado da taxa de câmbio futura, que pode levar ao overshooting, como descrito por Dornbusch (1976). Caso a política esteja associada a uma queda na credibilidade do país, os investimentos podem se tornar menos atrativos, reduzindo a entrada de capitais. Se a política estiver associada a uma restrição de crédito que reduza a oferta de bens exportados (considerando producer currency pricing) ${ }^{1}$, cai a demanda futura e, possivelmente, a demanda à vista de moeda, assim pode ocorrer uma depreciação. Assim, o efeito sobre a taxa de câmbio é ambíguo.

A segunda fonte de distorções na visão não convencional é sobre o retorno esperado. Furmam e Stiglitz (1998) destacam que não é a taxa de juros nominal em si que importa, mas sim o retorno esperado do investimento que está sujeito à probabilidade

\footnotetext{
1 Ver Engel (2000) e Devereux e Engel (2003) sobre como a escolha entre o local currency pricing e o producer currency pricing pode afetar a taxa de câmbio.
} 
de default. Por fim, o mercado pode ser avesso ao risco. Nesse caso, o anúncio de políticas contracionistas, que podem levar a períodos de recessão, afeta a percepção de risco dos agentes.

O debate sobre o efeito da política monetária na taxa de câmbio ganhou mais destaque com a crise asiática nos anos 1990. De acordo com Gould e Kamin (2000), muito foi discutido sobre a necessidade de elevar as taxas de juros como uma forma de estabilizar a moeda, reestabelecer a credibilidade e servir de base para dar início à recuperação econômica. No entanto, o argumento contrário à elevação se baseava na diminuição da capacidade dos tomadores de empréstimos honrarem suas dívidas, enfraquecendo o sistema bancário, reduzindo a confiança do investidor e levando a uma depreciação da moeda.

Para Stiglitz (1999), a resposta da taxa câmbio à política monetária é ambígua e depende de fatores circunstanciais, como períodos de crise, racionamento no mercado de crédito, desequilíbrios no balanço de pagamento, entre outros. Os resultados de estudos empíricos confirmam essa ambiguidade, sendo possível encontrar evidências sobre a validade da visão tradicional e também sobre falhas nessa ótica.

\subsection{O PROBLEMA DA ENDOGENEIDADE}

Segundo Caporale, Cipollini e Demetriades (2005), é necessário levar em consideração três fatores ao realizar trabalhos empíricos nesta área: a endogeneidade da política monetária; a orientação da política monetária, ou seja, se é expansionista ou contracionista; e a possibilidade de mudança de regimes.

O problema da endogeneidade entre a política monetária e a taxa de câmbio surge porque a taxa de juros de curto prazo influencia o comportamento da taxa de câmbio ao mesmo tempo em que é afetada por ela. De acordo com Kohlscheen (2014), a endogeneidade se dá pela possibilidade de que uma ação da política monetária, que afeta a taxa de câmbio, consiste em uma reação da política monetária aos movimentos na taxa de câmbio.

Segundo Taylor (2001), mesmo que um país adote taxa de câmbio flutuante, metas de inflação e um instrumento para política monetária, não há um modo efetivo de separar os movimentos da taxa de câmbio da taxa de juros. A pergunta que surge é sobre a postura da autoridade monetária: deveria o Banco Central do Brasil reagir aos movimentos da taxa de câmbio ou focar apenas nos indicadores domésticos?

Para ajudar a responder essa pergunta é preciso compreender os efeitos da taxa de câmbio nos preços. Além dos efeitos já citados sobre a demanda agregada e diretamente na inflação via bens comercializáveis e insumos importados, Taylor (2001) 
destaca um efeito indireto da taxa de câmbio na política monetária que surge devido à defasagem do processo de transmissão. De acordo com o autor, uma apreciação da taxa de câmbio hoje diminui as expectativas em relação ao produto interno bruto e a inflação futuros, criando uma expectativa de queda na taxa de juros futura.

Esse efeito ainda pode ser aumentado por dois fatores, um modelo de estrutura a termo da taxa de juros com expectativas racionais e uma regra de instrumento que considere o valor esperado das variáveis de interesse, inflação e produto. No primeiro caso, conforme Taylor (2001), uma queda no valor futuro esperado para a taxa de juros de curto prazo tende a diminuir as taxas de juros de longo prazo no presente, de forma que a apreciação da taxa de câmbio, mesmo que indiretamente, resulta em uma queda na taxa de juros no presente. Além disso, caso a regra para o instrumento da política monetária considere as expectativas futuras em relação à inflação e produto, a queda no valor esperado dessas variáveis faz com que o Banco Central reduza taxa de juros no futuro, mesmo que essa queda tenha sido ocasionada por uma apreciação da taxa de câmbio.

Dessa forma, como destacado por Taylor (2001), não é possível separar a taxa de câmbio da política monetária, sendo que, mesmo que a taxa de câmbio não apareça diretamente na regra do instrumento de política, seu efeito indireto sobre as expectativas de inflação e produto faz com que ela seja considerada implicitamente na determinação da taxa de juros.

Como exemplo prático,no caso brasileiro é possível analisar os Relatórios de Inflação, publicados trimestralmente pelo Banco Central, e as atas das reuniões do Comitê de Política Monetária (COPOM), também disponibilizadas pelo Banco Central. Em ambos os documentos são colocados cenários condicionantes para a projeção da inflação que fundamenta a decisão sobre a taxa de juros. Um desses cenários utiliza as expectativas do Boletim Focus em relação à trajetória da taxa de câmbio e da taxa básica de juros, a taxa Selic, enquanto que o outro cenário mantém constante essas variáveis. Embora um conjunto maior de variáveis seja utilizado para a decisão da meta para a taxa Selic, destaca-se que a taxa de câmbio também terá seu efeito na decisão através das projeções de inflação, além do seu impacto direto na demanda agregada.

Caso a origem da depreciação da moeda brasileira seja uma elevação do risco externo do país, expresso em maior retorno exigido em seus títulos, a elevação dos juros necessária ao controle da inflação gera a relação positiva entre depreciação da moeda e taxa de juros.

A endogeneidade da política monetária pode gerar um problema de causalidade reversa. De acordo com Zettelmeyer (2004), o modo como esse problema é tratado é capaz de influenciar o resultado do estudo. Para o autor, é possível inferir que, através da literatura existente, o efeito da política monetária sobre a taxa de câmbio é espúrio, dada a falha em controlar o problema da endogeneidade. 


\section{EVIDÊNCIAS EMPÍRICAS PARA O CASO BRASILEIRO}

Em relação ao caso específico do Brasil, Blanchard (2004) analisa a presença de dominância fiscal no país entre 2002 e 2003. O trabalho verifica como um ambiente de alto nível de endividamento e aversão ao risco pode levar a uma relação inversa entre a taxa de juros e a taxa de câmbio, especialmente no caso de um regime de metas de inflação, no qual o aumento na taxa de juros de curto prazo frente a pressões inflacionárias é a resposta mais comum da autoridade monetária. Nesse contexto, o aumento na taxa de juros pode agravar ainda mais a situação e, nesse caso, a política fiscal é um instrumento mais eficiente para o controle dos preços do que a política monetária. A preocupação de Blanchard (2004) é verificar sob quais condições um aumento na taxa real de juros pode levar a uma depreciação real da moeda, levando em consideração os efeitos da taxa real de juros na probabilidade de default da dívida pública. O autor utiliza um modelo que formaliza as relações entre a taxa de juros, taxa de câmbio e a probabilidade de default, considerando cenários com a dívida inicial elevada e também com alta aversão ao risco. São descritas três relações no trabalho. A primeira mostra a influência do risco de default e da taxa real de juros na taxa de câmbio, levando em consideração o grau de aversão ao risco dos investidores. A segunda relação descreve a interação entre a dívida pública e o risco de default. Por fim, a relação entre a probabilidade de default e o valor esperado da dívida.

Após descrever essas três relações, Blanchard (2004) apresenta duas equações que representam o modelo e são utilizadas para analisar o efeito da taxa de juros na taxa de câmbio e no risco default. O modelo é estimado considerando valores dados para a taxa de juros real doméstica $(r)$ e estrangeira $\left(r^{*}\right)$, nível total da dívida pública $(D)$, superávit primário $(X)$ e para os parâmetros para a relação dívida em dólar/dívida total $(\mu)$, aversão ao risco estrangeira $\left(\theta^{*}\right)$ e a parcela de aversão ao risco doméstica como função da aversão ao risco estrangeira $(\lambda)$.

Para estimar as relações, Blanchard (2004) utilizou mínimos quadrados ordinários, autoregressão de ordem $1(\mathrm{AR}(1))$ e um $\mathrm{AR}(1)$ com variáveis instrumentais. A partir desses resultados é finalmente estimado o efeito da taxa de juros na taxa de câmbio para diferentes níveis de $D, \mu$ e $\theta^{*}$. Os resultados mostram que um aumento de um ponto percentual na taxa Selic pode levar a até $8,57 \%$ de depreciação na taxa de câmbio considerando $D=0,63$. Já considerando $\mu=0,50$, a depreciação pode chegar a 2,58\%. Considerando $\theta^{*}=0,50$, a depreciação também é de $2,58 \%$. De modo geral, Blanchard (2004) mostra que o impacto da taxa de juros na taxa de câmbio depende muito das condições inicias. Quanto maiores os valores iniciais para a dívida total, dívida em dólar e aversão ao risco dos investidores estrangeiros, mais provável é que um aumento na taxa de juros leve a uma depreciação da taxa de câmbio. 
A dominância fiscal é frequentemente relacionada na literatura como causa da relação inversa entre a taxa de juros e a taxa de câmbio. Nesse sentido, os resultados apresentados por Blanchard (2004) corroboram com essa hipótese.

Gonçalves e Guimarães (2011) desenvolvem um modelo que leva em consideração a presença de dominância fiscal, porém utilizam a metodologia de identificação via hetorecedasticidade para lidar com o problema de causalidade reversa. Uma das vantagens apresentadas pelo trabalho é não focar apenas em períodos de crise, como muitos trabalhos que investigam a relação entre política monetária e taxa de câmbio fazem. Os autores formulam a hipótese de que a resposta da taxa de câmbio às variações na taxa de juros apresenta o formato em U: para valores baixos de dívida uma elevação na taxa de juros leva a uma apreciação da moeda, no entanto, quando o valor da dívida passa de um determinado limite a moeda local começa a depreciar.

Para verificar empiricamente a hipótese, Gonçalves e Guimarães (2011) estimam as seguintes equações a partir de dados semanais para o período entre janeiro de 2000 a dezembro de 2006:

$$
\begin{aligned}
& \Delta e t=\alpha \Delta i t+z t+\eta t \\
& \Delta i t=\beta \Delta e t+\gamma z t+\varepsilon t
\end{aligned}
$$

Em (1) e (2), $\Delta$ et é a variação da taxa de câmbio à vista, obtida como a diferença no logaritmo da taxa de câmbio à vista entre terça e quinta feira; $\Delta$ it é a variação na taxa de juros de um ano, obtida como a diferença no logaritmo de (1) mais a taxa de juros de um ano entre terça e quinta feira; $z t$ é uma variável omitida; $\varepsilon t$ é o choque de política monetária; e $\eta t$ é o choque da taxa de câmbio. A amostra é dividida em duas: a subamostra $\mathrm{C}$ compreende os dias em que houve encontro do COPOM, com tamanho TC; e a subamostra N para os demais dias, com o tamanho TN.

Para contornar o problema de variáveis omitidas e endogeneidade da política monetária Gonçalves e Guimarães (2011) utilizam a metodologia de identificação via heterocedasticidade, proposta por Rigobon e Sack (2004). Parte-se da suposição de que a variância do choque da taxa de juros ( $\varepsilon t$ ) é maior no grupo $\mathrm{C}$ do que a variância do choque da taxa de juros no grupo N. A intuição por trás dessa suposição é que em dias de reunião do COPOM espera-se um choque adicional nas taxas de juros do mercado.

Gonçalves e Guimarães (2011) estimam a nova relação entre taxa de juros e a taxa de câmbio a partir dos instrumentos baseados em Rigobon e Sack (2004) e os resultados mostram que um aumento inesperado de 100 pontos base (um ponto percentual) na taxa de juros de um ano leva a até $2 \%$ de depreciação na taxa de câmbio.

Kohlscheen (2014) estuda os impactos de um choque da política monetária em três países emergentes: Brasil, Chile e México. A abordagem utilizada no trabalho se- 
gue a linha de Zettelmeyer $(2000,2004)$ que utiliza um método de análise de eventos para evitar o problema de causalidade reversa da política monetária, comum em modelos VAR. O método utilizado por Kohlscheen (2014) apresenta vantagens em relação ao modelo original por levar em consideração algumas características importantes que não estão presentes em Zettelmeyer (2000, 2004), como a intervenção direta do Banco Central no mercado de câmbio. O modelo também não se restringe aos eventos que resultaram em mudança na taxa de juros. $\mathrm{O}$ autor compreende que a decisão de não alterar a taxa de juros também pode ser vista como um choque. A principal desvantagem dessa abordagem é que apenas o impacto imediato pode ser observado, não relevando informações sobre a dinâmica do ajuste da taxa de câmbio.

O período analisado por Kohlscheen (2014) compreende de janeiro de 2003 a maio de 2011, os dados são diários e obtidos com base nos encontros dos comitês de política monetária de cada país. O autor analisa o desenvolvimento das variáveis no dia seguinte de cada evento, lembrando que os anúncios são feitos após o fechamento do mercado, por isso a utilização do dia seguinte ao evento. Como proxy para surpresa monetária utiliza a taxa de juros do mercado, partindo da hipótese de que ela só é afetada por componentes não antecipados da política. No entanto, para checar a robustez o autor também utiliza a mudança da taxa de juros de curto prazo determinada pelo Banco Central e, no caso brasileiro, a diferença entre a meta anunciada e a expectativa de mercado sobre a meta.

Para verificar o impacto da política monetária na taxa de câmbio, Kohlscheen (2014) estima a equação (3):

$$
\Delta e t=\alpha+\beta \Delta i n, t+\gamma \Delta Z t+\varepsilon t
$$

Em (3), $\Delta i n, t$ é a variação da taxa de juros de n-meses; $\Delta e t$ é a variação diária na taxa de câmbio; $Z t$ a variação das possíveis variáveis de controle, no dia do anúncio ou no dia seguinte; $\varepsilon t$ é o termo de erro com média zero e variância constante; e $\alpha$ é incluída para captar algum tipo de tendência de depreciação. A inclusão de $Z t$ na equação (3) é uma inovação em relação ao modelo de Zettelmeyer (2000, 2004), que não considera possíveis variáveis de controle.

A equação (3) é estimada por mínimos quadrados ordinários (MQO) e pelo método de variáveis instrumentais (IV), utilizando a taxa de juros de curto prazo determinada pelo Banco Central como instrumento para a taxa de juros de mercado. As variáveis de controle utilizadas por Kohlscheen (2014) captam as variações nas condições monetárias estrangeiras, prêmio de risco e preço internacional das commodities.

Os resultados apontam para uma relação positiva entre a taxa de juros e a taxa de câmbio, ao contrário do que supõe a visão teórica tradicional. Um aumento de 100 pontos base na taxa de juros leva a uma depreciação da taxa de câmbio em cerca de 
$1 \%$ a 2,4\%, dependendo do país. As variáveis de controle apresentam o sinal esperado e as estimativas são robustas à exclusão dos eventos que coincidem com intervenções no mercado cambial.

Adicionalmente, Kohlscheen (2014) verifica a robustez das estimativas alternando as variáveis para taxa de câmbio e surpresa monetária, como a utilização da taxa de câmbio efetiva e a diferença entre a taxa Selic anunciada e a expectativa da taxa no boletim Focus, respectivamente, e conclui que não há mudanças qualitativas no modelo com as novas medidas. Também são excluídos eventos nos quais a taxa de juros de mercado variou menos de cinco pontos base no dia seguinte ao anúncio, assim como eventos que não resultaram em mudança na taxa básica de juros do governo. Os resultados mostram que esses eventos não são os responsáveis pelo sinal inverso na relação entre taxa de juros e taxa de câmbio.

Por fim, Kohlscheen (2014) verifica a presença de assimetria, separando a amostra em surpresas positivas e surpresas negativas. De acordo com o teste $t$ não é possível rejeitar a hipótese de que os coeficientes de inclinação são os mesmos nos dois casos. De acordo com Kohlscheen (2014), esse é mais um sinal de que os resultados não podem ser atribuídos à dominância fiscal. $\mathrm{O}$ primeiro sinal é a falta de evidência empírica significativa entre taxa de juros e risco de default; o segundo, a ausência de assimetria, o que significa que não é possível indicar a presença de efeitos não lineares, como pressupõe a hipótese da dominância fiscal.

Kohlscheen (2014) conclui com uma análise da literatura relacionada em busca de possíveis explicações para a relação não tradicional entre política monetária e taxa de câmbio. De acordo com o autor, atribuir esses resultados à causalidade reversa e à dominância fiscal não fornece uma explicação razoável para o exchange rate puzzle e sugere mais estudos sobre a relação entra a taxa de juros outras variáveis.

\section{METODOLOGIA E ANÁLISE EMPÍRICA}

\subsection{APRESENTAÇÃO DOS DADOS}

A estrutura de dados utilizados é similar a de Gonçalves e Guimarães (2011). O período analisado compreende janeiro de 2004 a janeiro de 2016, com observações diárias divididas em dois subgrupos: grupo $\mathrm{C}$, que corresponde a dias em que ocorreram encontros do Comitê de Política Monetária (COPOM); grupo N, que corresponde aos mesmos dias da semana em semanas sem encontro. A Tabela 1 apresenta as variáveis utilizadas e a Tabela 2 apresenta as suas estatísticas descritivas de todo o período e também para os dois grupos, COPOM e Não COPOM. 
Tabela 1 - Apresentação das variáveis

\begin{tabular}{ccc}
\hline Variável & Descrição & Fonte \\
\hline di_360 & Taxa referencial de swaps DI x pré - prazo de 360 dias, diária & BCB \\
selic & Taxa Selic anualizada base 252 & BCB \\
e & Taxa de câmbio, dólar comercial, compra & BCB \\
vix & volatility index & CBOE \\
Embi & EMBI+Br & IPEADATA \\
\hline
\end{tabular}

Nota: BCB - Banco Central do Brasil, sistema gerenciador de séries temporais. CBOE: Chicago Board Options Exchange. Fonte: Elaboração própria.

Nas estimações são utilizadas as variações entre o dia do anúncio e o dia seguinte, obtidas com as equações (4) a (7):

$$
\begin{aligned}
& \Delta e=\log \left(e_{\text {quinta- } f}\right)-\log \left(e_{\text {quarta- } f}\right) \\
& \Delta i=\log \left(1+i_{\text {quinta- } f}\right)-\log \left(1+i_{\text {quarta }-f}\right) \\
& \Delta v i x=\log \left(\text { vix }_{\text {quinta }-f}\right)-\log \left(\text { vix }_{\text {quarta }-f}\right) \\
& \Delta e m b i=\log \left(e m b i_{\text {quinta- } f}\right)-\log \left(e m b i_{\text {quarta-f }}\right)
\end{aligned}
$$

Nas equações (4) a (7), $\Delta e$ é a variação da taxa de câmbio; $\Delta i$ é a variação na taxa de juros; $\Delta$ vix é a variação do índice de volatilidade; e $\Delta e m b i$ é a variação do EMBI+Br. Como as reuniões do COPOM seguem uma agenda pública e as decisões são comunicadas às quartas-feiras, após o fechamento do mercado, optou-se por utilizar a variação entre quinta-feira e quarta-feira.

De acordo com Zettelmeyer (2000), é possível utilizar a variação de apenas um dia da taxa de juros e da taxa de câmbio porque elas se comportam como preços de ativos em mercados líquidos, ou seja, a resposta aos choques é imediata. Além de possibilitar o estudo do efeito de política monetária no curto prazo, antes do ajuste nas variáveis reais.

Como taxa de juros optou-se por utilizar a taxa de juros de um ano, assim como Gonçalves e Guimarães (2011). O prazo de um ano é longo o suficiente para captar apenas mudanças não esperadas na taxa de juros de curto prazo determinada pelo Banco Central, ao contrário das taxas com prazos mais curtos que apresentam mais ruído. A própria taxa Selic também é usada como instrumento na regressão para medida de um choque de política monetária.

O índice de volatilidade (VIX) é utilizado como uma medida de aversão ao risco dos investidores, um aumento no VIX significa um aumento na volatilidade do mercado. Utiliza-se também o Emerging Markets Bond Index Plus (EMBI+), que reflete os retornos diários de uma carteira composta por títulos de dívida emitidos por países emergentes. Utiliza-se o spread do $\mathrm{EMBI}+\mathrm{Br}$ como medida para o risco-Brasil. $\mathrm{O}$ spread é obtido pela diferença entre a taxa de retorno de títulos da dívida brasileira 
em relação aos títulos de prazo equivalente do Tesouro dos Estados Unidos, que são considerados livres de risco. Quanto maior o EMBI+, maior o risco do país.

Tabela 2 - Estatísticas descritivas das variáveis*

\begin{tabular}{ccccccc}
\hline & Média & Desvio padrão & $\begin{array}{c}\text { Coeficiente de } \\
\text { variação }\end{array}$ & Mínimo & Máximo & $\mathbf{n}^{\mathbf{o}}$ obs \\
\hline taxa de câmbio & 2,2184 & 0,5262 & $24 \%$ & 1,5559 & 4,1942 & 620 \\
Selic & 12,3205 & 3,2982 & $27 \%$ & 7,11 & 19,76 & 620 \\
di_360 & 12,5536 & 3,0938 & $25 \%$ & 6,86 & 19,24 & 620 \\
Vix & 19,3818 & 9,3712 & $48 \%$ & 10,05 & 79,13 & 620 \\
Embi & 277,9918 & 121,7739 & $44 \%$ & 137 & 763 & 620 \\
\hline \multicolumn{7}{c}{ Subamostra: Não COPOM } \\
\hline taxa de câmbio & 2,2099 & 0,5193 & $23 \%$ & 1,5573 & 4,1942 & 516 \\
Selic & 12,2241 & 3,2295 & $26 \%$ & 7,11 & 19,76 & 516 \\
di_360 & 12,4669 & 3,0291 & $24 \%$ & 6,86 & 19,19 & 516 \\
\hline taxa de câmbio & 2,2739 & Subamostra: COPOM & & & 104 \\
Selic & 12,6855 & 0,5616 & $25 \%$ & 1,5559 & 4,1552 & 104 \\
di_360 & 12,8913 & 3,4709 & $27 \%$ & 7,11 & 19,76 & 104 \\
\hline
\end{tabular}

Nota: *em nível.

Fonte: Elaboração própria.

A Tabela 3 apresenta os resultados para o teste de Mann-Whitney para verificar se os dados das subamostras para os dias com reunião do COPOM e sem reunião do COPOM pertencem à mesma população. Ao contrário do teste $t$, o teste de Mann-Whitney não necessita das hipóteses de normalidade ou homogeneidade das variâncias, além de permitir testar amostras com tamanhos diferentes.

O teste de Mann-Whitney consiste na ordenação de todas as variáveis das duas amostras (A e B) em um único grupo, de forma crescente. A ordem de cada elemento é registrada e as amostras são novamente separadas. Calcula-se o número de vezes em que as observações da amostra $A$ foi maior do que as observações da amostra $B$, que pode ser chamado de $\mathrm{U}_{\mathrm{A}}$, obtém-se $\mathrm{U}_{\mathrm{B}}$ de maneira análoga. Quanto menor o valor de $\mathrm{U}_{\mathrm{A}}$ maior a evidência de que as populações são distintas. Utiliza-se então o menor valor de U como estatística do teste. Para amostras pequenas, até 20 observações, existe uma distribuição teórica exata do teste e para amostras grandes utiliza-se a distribuição normal como aproximação.

Tabela 3 - Teste de Mann-Whitney

\begin{tabular}{ccc}
\hline Variável & $\mathbf{U}$ & P valor \\
\hline$\Delta \mathrm{e}$ & 1,3959 & 0,06127 \\
$\Delta$ di_360 & 1,0687 & 0,2852 \\
$\Delta$ selic & 0,1591 & 0,8735 \\
$\Delta$ vix & 0,4997 & 0,6173 \\
$\Delta$ embi & 1,5402 & 0,1235 \\
\hline
\end{tabular}

Fonte: Elaboração própria. 
O teste de Mann-Whitney tem como hipótese nula a igualdade de população entre as amostras que pertencem à mesma população. Como é possível verificar através da Tabela 3, a hipótese nula só pode ser rejeitada para as variações da taxa de câmbio, considerando um nível de significância de $10 \%$. Esse resultado indica que há uma mudança no comportamento da taxa de câmbio em dias de COPOM. Em relação às demais variáveis, não é possível rejeitar a hipótese nula.

\subsection{METODOLOGIA}

Para estimar os efeitos de uma variação na taxa de juros sobre a taxa de câmbio, é preciso levar em consideração a presença de endogeneidade e a existência de variáveis omitidas. De acordo com Rigobon e Sack (2004), essas características podem ser captadas através do sistema de equações (1) e (2).

Dada a presença de equações simultâneas e variável omitida, as equações (1) e (2) não podem ser estimadas consistentemente através de MQO. Considere aplicar o MQO na equação (1), o coeficiente a será viesado porque o choque $\eta_{t}$ é correlacionado com o regressor $\Delta i_{t}$, ou seja, caso $\beta \neq 0$ e $\sigma_{\eta}>0$, teremos um viés por causa da endogeneidade de $\Delta i_{t}$. Já a omissão de variável gera um viés que depende do valor de $\gamma$. Ou seja, caso $\gamma \neq 0$ e $\sigma_{\gamma}>0$ teremos um viés de variável omitida.

A abordagem de estudos de eventos, que se concentra em analisar o comportamento das variáveis em períodos que cercam as mudanças no instrumento de política monetária, é empregada como uma forma de evitar o problema de endogeneidade. No entanto, de acordo com Rigobon (2003), para que a estimativa de a não seja viesada é necessário impor algum tipo de restrição sobre os parâmetros das equações (1) e (2). As restrições podem ser: restrições de exclusão, ou seja, assumir que $\beta=0$ ou $\alpha=0$; restrições de sinais dos parâmetros; restrições de longo prazo, que inclui variáveis defasadas no sistema; e restrições de variância, $\sigma_{\varepsilon} /\left(\sigma_{\eta} \rightarrow \infty\right)$ e $\sigma_{\varepsilon} /\left(\sigma_{z} \rightarrow \infty\right)$.

De acordo com o autor, embora úteis, essas restrições podem não ser racionais no contexto econômico. A abordagem de identificação via heterocedasticidade parte da suposição de que há dois regimes na variância dos choques, alta volatilidade e baixa volatilidade. Não é necessário assumir que a variância dos choques se torna infinita, é preciso apenas que haja um aumento na variância dos choques relativo aos demais períodos.

No caso específico deste trabalho, considera-se que a variância do choque de política monetária $\left(\varepsilon_{t}\right)$ é maior em dias de encontro do COPOM, como pode ser observado na tabela 2 . Nesses dias, grande parte das notícias que afetam o mercado é relacionada à decisão da política monetária. As demais variâncias, $\eta_{t}$ e $z_{t}$, são consideradas constantes e os parâmetros estáveis: 


$$
\begin{aligned}
& \sigma_{\varepsilon}^{C}>\sigma_{\varepsilon}^{N} \\
& \sigma_{\eta}^{C}>\sigma_{\eta}^{N} \\
& \sigma_{\mathrm{z}}^{C}>\sigma_{\mathrm{z}}^{N}
\end{aligned}
$$

O sobrescrito $\mathrm{C}$ indica que as observações pertencem ao grupo $C$, ou seja, dias de encontro do COPOM, e o sobrescrito $N$ indica as observações pertencentes ao grupo $\mathrm{N}$, que compreende os demais dias. Também é considerado que os choques não possuem correlação serial e não são correlacionados entre si, ou com o choque em comum $z_{t}$.

Analiticamente a identificação utilizando heterocedasticidade pode ser vista a partir da resolução da forma reduzidas das equações (1) e (2), como mostram Rigobon e Sack (2004) e Gonçalves e Guimarães (2011):

$$
\begin{aligned}
\Delta i_{t} & =\frac{1}{1-\alpha \beta}\left[(\beta+\gamma) z_{t}+\beta_{\eta t}+\varepsilon_{t}\right] \\
\Delta \mathrm{e}_{t} & =\frac{1}{1-\alpha \beta}\left[(1+\alpha \gamma) z_{t}+\eta_{t}+\alpha \varepsilon_{t}\right]
\end{aligned}
$$

A matriz de covariância para cada subamostra é dada por $\Omega_{C}=E\left[\left[\Delta i_{t} \Delta e_{t}\right]^{\prime} \cdot\left[\Delta i_{t}\right.\right.$ $\left.\left.\Delta e_{t}\right] \mid t \in C\right]$ e $\Omega_{N}=E\left[\left[\Delta i_{t} \Delta e_{t}\right]^{\prime} \cdot\left[\Delta i_{t} \Delta e_{t}\right] \mid t \in N\right]$. As matrizes são determinadas por:

$$
\begin{aligned}
& \Omega_{C}=\frac{1}{(1-\alpha \beta)^{2}}\left[\begin{array}{cc}
\sigma_{\varepsilon}^{C}+\beta^{2} \sigma_{\eta}^{C}+(\beta+\gamma)^{2} \sigma_{z}^{C} & \alpha \sigma_{\varepsilon}^{C}+\beta \sigma_{\eta}^{C}+(\beta+\gamma)(1+\alpha \gamma) \sigma_{z}^{C} \\
\cdot & \alpha^{2} \sigma_{\varepsilon}^{C}+\beta^{2} \sigma_{\eta}^{C}+(1+\alpha \gamma)^{2} \sigma_{z}^{C}
\end{array}\right] \\
& \Omega_{N}=\frac{1}{(1-\alpha \beta)^{2}}\left[\begin{array}{cc}
\sigma_{\varepsilon}^{N}+\beta^{2} \sigma_{\eta}^{N}+(\beta+\gamma)^{2} \sigma_{z}^{N} & \alpha \sigma_{\varepsilon}^{N}+\beta \sigma_{\eta}^{N}+(\beta+\gamma)(1+\alpha \gamma) \sigma_{z}^{N} \\
\cdot & \alpha^{2} \sigma_{\varepsilon}^{N}+\beta^{2} \sigma_{\eta}^{N}+(1+\alpha \gamma)^{2} \sigma_{z}^{N}
\end{array}\right]
\end{aligned}
$$

Realizando a diferença entre as duas matrizes tem-se:

$$
\Delta \Omega \equiv \Omega_{C}-\Omega_{N}=\frac{\left(\sigma_{\varepsilon}^{C}-\sigma_{\varepsilon}^{N}\right)}{(1-\alpha \beta)^{2}}\left[\begin{array}{cc}
1 & \alpha \\
\alpha & \alpha^{2}
\end{array}\right]
$$

É possível identificar $\alpha$ a partir da diferença entre as matrizes de covariância de duas maneiras:

$$
\alpha_{\text {het }}=\frac{\Delta \Omega_{12}}{\Delta \Omega_{11}}
$$




$$
\alpha_{\text {het }}=\frac{\Delta \Omega_{22}}{\Delta \Omega_{12}}
$$

Nas equações (14) e (15), $\alpha_{\text {het }}$ é o estimador baseado na heterocedasticidade e $\Delta \Omega_{\mathrm{ij}}$ representa o elemento $(i, j)$ da diferença da matriz $\Omega$. De acordo com Rigobon e Sack (2004), esses estimadores se assemelham ao estimador dos modelos de estudos de evento, que, neste caso, é o aumento da variância dos choques em dias de COPOM. Caso todas as hipóteses do modelo sejam válidas as estimativas de $\alpha$ serão iguais ${ }^{2}$.

Para implementar o método de variáveis instrumentais Rigobon e Sack (2004) definem as seguintes variáveis:

$$
\begin{aligned}
& \Delta I \equiv\left[\begin{array}{ll}
\frac{\Delta i_{C}^{\prime}}{\sqrt{T_{C}}} & \frac{\Delta i_{N}^{\prime}}{\sqrt{T_{N}}}
\end{array}\right]^{\prime} \\
& \Delta E \equiv\left[\begin{array}{ll}
\frac{\Delta e_{C}^{\prime}}{\sqrt{T_{C}}} & \frac{\Delta e_{N}^{\prime}}{\sqrt{T_{N}}}
\end{array}\right]^{\prime} \\
& w_{i} \equiv\left[\frac{\Delta i_{C}^{\prime}}{\sqrt{T_{C}}}-\frac{\Delta i_{N}^{\prime}}{\sqrt{T_{N}}}\right]^{\prime} \\
& w_{s} \equiv\left[\begin{array}{ll}
\frac{\Delta e_{C}^{\prime}}{\sqrt{T_{C}}} & \frac{\Delta e_{N}^{\prime}}{\sqrt{T_{N}}}
\end{array}\right]
\end{aligned}
$$

Nas equações (16) a (19), Tc e Tn são os tamanhos das amostras $C$ e $N$, respectivamente, as variáveis $w_{i}$ e $w_{s}$ serão utilizadas como instrumentos. A validade dessas variáveis como instrumentos está relacionada, intuitivamente, com a correlação que existe entre $w_{i}$ e $\Delta i$, como a variância em dias de COPOM é maior a correlação entre

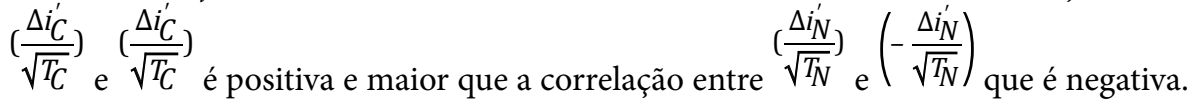
$\mathrm{O}$ mesmo raciocínio pode ser feito para o $w_{s}$ e $\Delta E$. Formalmente, para o caso do instrumento $w_{i}$, o raciocínio é análogo para $w_{s}:^{3}$

$$
\begin{aligned}
& \operatorname{plim} \frac{1}{T} w_{i}^{\prime} \Delta \mathrm{I}=\frac{1}{T_{C}} \Delta \mathrm{i}_{C}^{\prime} \cdot \Delta i_{C}-\frac{1}{\mathrm{~T}_{\mathrm{N}}} \Delta i_{N}^{\prime} \cdot \Delta i_{N} \\
& =\frac{(\beta+\gamma)^{2}}{(1-\alpha \beta)^{2}} \sigma_{\varepsilon}^{C}-\frac{(\beta+\gamma)^{2}}{(1-\alpha \beta)^{2}} \sigma_{\varepsilon}^{C}>0
\end{aligned}
$$

2 Para prova desses resultados ver Rigobon (2003).

3 Ver Rigobon e Sack (2004) para a derivação completa dos estimadores e também suas propriedades. 
Tem-se que $w_{i}$ também não será correlacionado $\operatorname{com} z$ e com $\eta$ :

$$
\begin{aligned}
& p \operatorname{plim} \frac{1}{T} w_{i}^{\prime} z=\frac{1}{T_{C}} \Delta \mathrm{i}_{C}^{\prime} \cdot z_{C}-\frac{1}{T_{N}} \Delta i_{N}^{\prime} \cdot z_{N} \\
& =\frac{(\beta+\gamma)^{2}}{(1-\alpha \beta)^{2}} \sigma_{z}^{C}-\frac{(\beta+\gamma)^{2}}{(1-\alpha \beta)^{2}} \sigma_{z}^{C}=0 \\
& p \operatorname{plim} \frac{1}{T} w_{i}^{\prime} \eta=\frac{1}{T_{C}} \Delta \mathrm{i}_{C}^{\prime} \cdot \eta_{C}-\frac{1}{T_{N}} \Delta i_{N}^{\prime} \cdot \eta_{N} \\
& =\frac{(\beta+\gamma)^{2}}{(1-\alpha \beta)^{2}} \sigma_{\eta}^{C}-\frac{(\beta+\gamma)^{2}}{(1-\alpha \beta)^{2}} \sigma_{\eta}^{C}=0
\end{aligned}
$$

A estimativa de $\alpha$ pode ser obtida da seguinte maneira:

$$
\begin{aligned}
& \hat{\alpha}_{\text {het }}^{i}=\left(w_{i}^{\prime} \Delta I\right)^{-1}\left(w_{i}^{\prime} \Delta E\right) \\
& \hat{\alpha}_{\text {het }}^{s}=\left(w_{s}^{\prime} \Delta I\right)^{-1}\left(w_{i}^{\prime} \Delta E\right)
\end{aligned}
$$

Que podem ser escritos como:

$$
\begin{aligned}
& \hat{\alpha}_{\text {het }}^{i}=\frac{\left\{\Delta i_{C},-\Delta i_{N}\right\}^{\prime}\left\{\Delta e_{C}, \Delta e_{N}\right\}}{\left\{\Delta i_{C},-\Delta i_{N}\right\}^{\prime}\left\{\Delta i_{C}, \Delta i_{N}\right\}}=\frac{\operatorname{Cov}\left(\Delta i_{C}, \Delta e_{C}\right)-\operatorname{Cov}\left(\Delta i_{N}, \Delta e_{N}\right)}{\operatorname{Var}\left(\Delta i_{C}\right)-\operatorname{Var}\left(\Delta i_{N}\right)} \\
& \hat{\alpha}_{\text {het }}^{e}=\frac{\left\{\Delta e_{C},-\Delta e_{N}\right\}^{\prime}\left\{\Delta e_{C}, \Delta e_{N}\right\}}{\left\{\Delta e_{C}-\Delta e_{N}\right\}^{\prime}\left\{\Delta i_{C}, \Delta i_{N}\right\}}=\frac{\operatorname{Var}\left(\Delta e_{C}\right)-\operatorname{Var}\left(\Delta i_{N}\right)}{\operatorname{Cov}\left(\Delta i_{C}, \Delta e_{C}\right)-\operatorname{Cov}\left(\Delta i_{N}, \Delta e_{N}\right)}
\end{aligned}
$$

Os estimadores de (24) e (25) são os mesmos das equações (18) e (19). Assim, o instrumento $w_{i}$ é considerado um instrumento válido para $\Delta I$, assim como $w_{s}$ é válido para $\Delta E$.

\subsection{HIPÓTESE DE IDENTIFICAÇÃO}

Antes de prosseguir com a estimação é necessário verificar a hipótese principal do método de identificação via heterocedasticidade, ou seja, é preciso verificar se há uma diferença entre as variâncias do grupo $\mathrm{C}$ e do grupo $\mathrm{N}$ : 


$$
\begin{aligned}
& \operatorname{Var}\left(\Delta i_{C}\right)-\operatorname{Var}\left(\Delta i_{N}\right)=\frac{\sigma_{\varepsilon}^{C}-\sigma_{\varepsilon}^{N}}{(1-\alpha \beta)^{2}}>0 \\
& \operatorname{Var}\left(\Delta e_{C}\right)-\operatorname{Var}\left(\Delta e_{N}\right)=\alpha^{2} \frac{\sigma_{\varepsilon}^{C}-\sigma_{\varepsilon}^{N}}{(1-\alpha \beta)^{2}}>0
\end{aligned}
$$

De acordo com Gonçalves e Guimarães (2011), tanto a variância de $\Delta i$ quanto de $\Delta e$ devem aumentar em dias de COPOM. No entanto, é esperado que o aumento da variância em $\Delta i$ seja maior em relação a $\Delta e$, visto que a variância de $\Delta e$ já é grande. Para testar essas hipóteses foi realizado um teste $\mathrm{F}$ de igualdade de variância entre as duas amostras.

Como é possível observar a partir da tabela 4, não é possível rejeitar a hipótese nula de que a variância de $\Delta e$ é a mesma para as duas amostras. Já para a variância de $\Delta i$ é possível rejeitar a hipótese nula de igualdade nas variâncias. Esses resultados permitem prosseguir com o método de estimação via heterocedasticidade, visto que a

\begin{tabular}{|c|c|c|}
\hline & $\operatorname{Var}(\Delta i)$ & $\operatorname{Var}(\Delta e)$ \\
\hline COPOM & $4,85 \mathrm{E}-07$ & $1,84 \mathrm{E}-05$ \\
\hline Não COPOM & $2,96 \mathrm{E}-05$ & $1,83 \mathrm{E}-05$ \\
\hline Diferença & $3,08 \mathrm{E}-07$ & $-1,55 \mathrm{E}-07$ \\
\hline Variação \% & 273,84 & 100,85 \\
\hline F - Calculado & 2,7384 & 1,0085 \\
\hline $\mathrm{F}-$ crítico $^{*}$ & 1,27 & 1,2698 \\
\hline p-valor & $1,02 \mathrm{E}-13$ & 0,4643 \\
\hline
\end{tabular}
diferença entre a variância de $\Delta i_{C}$ e $\Delta i_{N}$ é estatisticamente significativa.

\subsection{RESULTADOS}

Para a estimação são empregados os métodos de variáveis instrumentais (IV) e o método generalizado dos momentos (GMM). A variável dependente é descrita pela equação (19), o regressor é descrito pela equação (18) e os instrumentos são apresentados nas equações (20) e (21). O parâmetro de interesse é o a da equação (8). Os resultados são apresentados na Tabela 5. 


\begin{tabular}{|c|c|c|c|}
\hline & IV $-w_{i}$ & GMM $-w_{i}$ & GMM - $w_{i}, w_{s}$ \\
\hline \multirow{2}{*}{ di360 } & 1,5357 & 1,1545 & 1,1392 \\
\hline & $(0,000)$ & $(0,0196)$ & $(0,0198)$ \\
\hline $\mathrm{R}^{2}$ & 0,0291 & 0,0392 & 0,0668 \\
\hline het-test & $\begin{array}{c}0,2296 \\
(0,6324)\end{array}$ & - & - \\
\hline LM-BG & $\begin{array}{c}2,6469 \\
(0,2662)\end{array}$ & - & - \\
\hline \multirow{2}{*}{ Estatística J } & 1,9775 & 2,3039 & 5,0373 \\
\hline & $(0,1596)$ & $(0,316)$ & $(0,169)$ \\
\hline
\end{tabular}

Notas: p-valores entre parênteses.

Fonte: Elaboração própria.

Em relação aos resultados, os coeficientes obtidos por meio do método de IV são maiores e com um desvio padrão semelhante aos desvios estimados por GMM. Os coeficientes para a variável di360 são positivos e significativos estatisticamente nos três casos, havendo uma melhora no coeficiente de determinação com a estimação por GMM e utilizando ambos os instrumentos. Além disso, foram realizados os testes: Breusch-Pagan-Godfrey, que tem como hipótese nula a homocedasticidade; e o Breusch-Godfrey, que tem como hipótese nula a ausência de correlação serial. Não foi possível rejeitar a hipótese nula em ambos os testes.

Para verificar a validade dos instrumentos utilizou-se o teste de Sargan, que tem como hipótese nula a validade dos instrumentos. A partir dos $\mathrm{p}$-valores apresentados na tabela 4 para o teste, pode-se concluir que $w_{i}$ e $w_{s}$ são instrumentos válidos para a regressão, ou seja, os instrumentos não estão correlacionados com o termo de erro. $\mathrm{O}$ valor de a relacionado à variável di360 indica uma relação positiva entre as variações da taxa de juros e variações da taxa de câmbio. Ou seja, um aumento na taxa de juros leva a uma depreciação na taxa de câmbio, assim como previsto pela ótica revisionista. Os valores obtidos aqui são bastante semelhantes aos de Kohlscheen (2014) através de um estudo de eventos. Para dias sem intervenção no mercado monetário, o autor estima que uma variação da taxa de juros de três meses é responsável por 1,46\% de variação na taxa de câmbio. Já o nosso coeficiente estimado por meio do método de IV mostra que a taxa de câmbio se deprecia em aproximadamente 1,5\%, dada uma variação de um ponto percentual na taxa de juros de um ano. Enquanto Gonçalves e Guimarães (2011) estimam que a depreciação de aproximadamente 1,1\%, próximo dos resultados obtidos por GMM, 1,15\% e 1,13\%.

Comparando os resultados da tabela 6 com os resultados apresentados por Gonçalves e Guimarães (2011), é possível notar algumas diferenças. A inclusão dos instrumentos $w_{i}$ e $w_{s}$ na regressão de Gonçalves e Guimarães (2011) não produziu muita alteração nas estimativas entre o MQO e o IV. Os autores justificam esse resultado por 
meio da variância de $\Delta i$, no período analisado pelo trabalho a diferença observada na variância entre $\Delta i_{C}$ e $\Delta i_{N}$ foi $69,4 \%$, assim o instrumento $w_{i}$ não é fortemente correlacionado com $\Delta i$. Já a partir dos dados deste trabalho, a variância de $\Delta i_{C}$ é mais que o dobro de $\Delta i_{N}$, o que indica uma correlação mais forte entre o $w_{i}$ e $\Delta i$.

Essa diferença significativa na variância entre as duas amostras está relacionada ao período de análise. Nos últimos dez anos o Brasil passou por momentos de estabilidade e de crise. Os períodos de crise, como 2008, interromperam ciclos de queda na taxa Selic. Por exemplo, em março de 2008 a meta para taxa Selic era 11,25\%, em setembro a meta já estava em 13,75\%. Menos de um ano depois, em julho de 2009, a taxa Selic batia recorde de menor nível desde a adoção do regime de metas de inflação, $8,75 \%$. Este recorde foi superado em outubro de 2012, quando o COPOM anunciou a meta de $7,25 \%$.

Essas alterações constantes na taxa básica de juros ocorrem no Brasil porque o Banco Central utiliza o regime de metas de inflação e tem como principal instrumento a taxa básica de juros. Nesse contexto, pressões inflacionárias devem ser combatidas com mudanças na taxa de juros. Mudanças na postura do Banco Central nos últimos anos também podem estar relacionadas com a maior variabilidade das taxas de juros (cfe. ARAGÓN E PORTUGAL, 2009; CURADO e CURADO, 2014; PALMA e PORTUGAL, 2014; entre outros).

Os resultados apresentados na tabela 5 levam em consideração apenas a taxa de câmbio e a taxa de juros, sendo difícil fazer suposições sobre a origem dessa relação inversa entre essas variáveis. Portanto é necessário incluir outras variáveis para verificar a robustez dos resultados.

\subsection{ROBUSTEZ}

Para verificar a robustez das estimativas o modelo é reestimado com pequenas alterações: substituição da taxa de juros de um ano pela própria taxa Selic, inclusão de variáveis exógenas e uma medida de risco.

\subsubsection{TAXA SELIC}

Utilizar a taxa básica da política monetária no lugar da taxa de juros do mercado é um procedimento comum na literatura (ZETTELMEYER, 2000; HNATKOVSKA, LAHIRI e VEGH, 2011; KOHLSCHEEN, 2014; entre outros). Acredita-se que utilizar o próprio instrumento de política monetária como medida de choque elimina boa 
parte dos ruídos causados por outras variáveis na taxa de juros de um ano. A tabela 6 apresenta os resultados utilizando a taxa Selic no lugar da taxa di360. Outra alteração feita é que, para o grupo com observações dos dias do COPOM, utilizou-se a diferença entre a meta anunciada e a expectativa do mercado em relação à meta, para os demais dias permanece a variação entre quinta e quarta-feira da taxa Selic observada. Essa alteração é feita com o intuito de isolar apenas as surpresas na taxa Selic.

\begin{tabular}{|c|c|c|c|}
\hline & IV $-w_{i}$ & GMM $-w_{i}$ & GMM $-w_{i}, w_{s}$ \\
\hline \multirow{2}{*}{ Selic } & 0,1850 & 0,1827 & 0,1816 \\
\hline & $(0,094)$ & $(0,1825)$ & $(0,1888)$ \\
\hline $\mathrm{R}^{2}$ & 0,027 & 0,0273 & 0,0273 \\
\hline het-test & $\begin{array}{c}1,7254 \\
(0,1889)\end{array}$ & - & - \\
\hline LM-BG & $\begin{array}{c}3,3345 \\
(0,1888)\end{array}$ & - & - \\
\hline Estatística J & $\begin{array}{c}1,7796 \\
(0,6193)\end{array}$ & $\begin{array}{c}0,0219 \\
(0,8821)\end{array}$ & $\begin{array}{c}0,0752 \\
(0,7838)\end{array}$ \\
\hline
\end{tabular}

Notas: $p$-valores entre parênteses.

Fonte: Elaboração própria.

Como é possível observar, ao utilizar a taxa Selic e a diferença entre a meta anunciada e a expectativa da meta, os coeficientes não são estatisticamente significativos. Uma das possíveis explicações para esse resultado é que as expectativas do mercado brasileiro funcionam como um bom guia para a realização das variáveis, como destacado em Carvalho e Minella (2012). Entre 2004 e 2015 houve 103 reuniões do COPOM, considerando a mediana das expectativas do mercado para a taxa de juros do boletim Focus da semana anterior às reuniões, em apenas 29 dessas reuniões a decisão sobre a taxa de juros foi diferente da expectativa do mercado na semana anterior à reunião.

Em relação ao componente surpresa da política monetária, Zettelmeyer (2000) destaca que os comitês de política monetária que seguem uma agenda pública de encontros diminuem o fator surpresa. No caso brasileiro, o COPOM segue uma agenda pública e o anúncio sobre as medidas ocorre às quartas-feiras. Isto significa que mercado já espera alguma mudança em dias de COPOM e, como esses dias são divulgados com antecedência, as expectativas sobre as possíveis mudanças já são incorporadas pelos agentes, o que diminui a surpresa. Kohlscheen (2014) também testa uma variação do seu modelo com a substituição entre a taxa de juros de mercado de três meses por surpresas na meta da taxa Selic e não encontra uma relação significativa entre as surpresas e a taxa de câmbio.

Além disso, esse resultado também corrobora com a suposição feita por Gonçalves e Guimarães (2011), de que a taxa de câmbio não é diretamente afetada pelas decisões 
de política monetária. Por outro lado, percebe-se que a taxa de juros de um ano é diretamente afetada pelos encontros do COPOM, o que ocorre por meio da estrutura a termo da taxa de juros, enquanto que a taxa de câmbio só é afetada por influência da variação nessas taxas de juros de mercado, no caso deste trabalho, a taxa di360.

\subsubsection{INCLUSÃO DE REGRESSORES}

Incluem-se as variáveis: índice de volatilidade e EMBI+br. O VIX é incluído como uma variável exógena, uma vez que as decisões de política monetária brasileira não são capazes de afetar o índice, que reflete a aversão ao risco no mercado financeiro global (COUDERT e GEX, 2008; ESPINOZA e SEGOVIANO, 2011). Já o EMBI+Br é incluído como uma medida de risco-país, já que muitos trabalhos atribuem os resultados da visão revisionista ao aumento na probabilidade do risco de default (e.g. HILSCHER e NOSBUSCH, 2010). Os resultados são apresentados na tabela 7.

Tabela 7 - Resultados da regressão - Variável dependente $\Delta \mathrm{E}$

\begin{tabular}{cccc}
\hline & IV $-\boldsymbol{w}_{\boldsymbol{i}}$ & GMM $-\boldsymbol{w}_{\boldsymbol{i}}$ & GMM $-\boldsymbol{w}_{\boldsymbol{i}}, \boldsymbol{w}_{\boldsymbol{s}}$ \\
\hline \multirow{2}{*}{ di360 } & 1,4765 & 1,897 & 1,9501 \\
& $(0,0505)$ & $(0,0028)$ & $(0,0031)$ \\
Vix & 0,0110 & 0,0003 & 0,0006 \\
& $(0,1798)$ & $(0,1588)$ & $(0,4627)$ \\
Embi & 0,0148 & 0,0031 & 0,0652 \\
& $(0,0197)$ & $(0,0000)$ & $(0,0000)$ \\
$\mathrm{R}^{2}$ & 0,0595 & 0,056 & 0,1069 \\
\multirow{2}{*}{ het-test } & 1,3312 & & - \\
& $(0,7367)$ & - & - \\
LM-BG & 1,7183 & & 2,5807 \\
& $(0,1253)$ & - & $(0,1081)$ \\
\hline \multirow{2}{*}{ Estatística J } & 0,7194 & 2,1794 & $(0,1398)$ \\
\hline
\end{tabular}

Nota: p-valores entre parênteses.

Fonte: Elaboração própria.

É possível verificar que a taxa de juros e o EMBI+Br foram significativos nos três modelos e a variável VIX não apresentou coeficiente significativo em nenhum dos casos. Além disso, verifica-se que os sinais são conforme o esperado. O EMBI+Br também possui uma relação com a taxa de câmbio, assim temos que um aumento no risco país leva a uma depreciação da taxa de câmbio.

A inclusão de variáveis que captam a percepção de risco dos agentes é importante para verificar a hipótese sobre a dominância fiscal. De acordo com Kohlscheen (2014), é comum atribuir os resultados da ótica revisionista ao risco de default, porém nem 
sempre a relação entre taxa de câmbio, taxa de juros e risco é apresentada por autores que consideram essa hipótese. Os resultados apresentados, principalmente na tabela 7, permitem inferir que as variações da taxa de câmbio são influenciadas por variações no risco-país. No entanto, não é possível concluir que essa relação é a responsável pelos resultados da ótica revisionista apenas com os resultados aqui apresentados.

\section{CONSIDERAÇÕES FINAIS}

O debate sobre a relação entre a política monetária e a taxa de câmbio ainda está longe de um consenso. De acordo com a teoria, um aumento na taxa de juros leva a uma apreciação da taxa de câmbio, mas as evidências empíricas são mistas, um aumento na taxa de juros pode estar associado tanto a uma apreciação quanto a uma depreciação na taxa de câmbio.

O principal problema dos trabalhos empíricos é conseguir controlar a endogeneidade da política monetária. Porém, não há um modelo padrão para esse problema, autores que utilizaram a mesma abordagem encontram resultados distintos, como Zettelmeyer (2000) e Kohlscheen (2014).

O presente trabalho se baseou na abordagem utilizada por Gonçalves e Guimarães (2011) que utiliza o método de identificação via heterocedasticidade proposto por Rigobon (2003) e Rigobon e Sack (2004). Os resultados apresentados corroboram com a ótica revisionista, ou seja, há uma relação positiva entre as variações da taxa de câmbio e as variações da taxa de juros de mercado de um ano, que é utilizada como medida de política, assim como Gonçalves e Guimarães (2011).

Porém, o período analisado permite realizar algumas extensões importantes. Primeiro, verifica-se que a troca entre a taxa de juros de mercado pela taxa básica da política monetária não produz resultados significativos. Podemos inferir a partir desse resultado que a taxa de câmbio não responde diretamente às variações na taxa Selic. A mudança na taxa básica de juros faz variar a taxa de juros de mercado de um ano, por intermédio da estrutura a termo da taxa de juros, e são as variações da taxa de juros de mercado que terão efeito na variação da taxa de câmbio.

Outra extensão importante é a inclusão de variáveis que captam a percepção de risco dos agentes e também do risco-país. É comum atribuir o resultado revisionista à probabilidade de default, embora nem todos os trabalhos apresentem evidências para essa hipótese. Nesse caso, os resultados mostram que há uma relação positiva entre o risco e a variação na taxa de câmbio, porém não é possível concluir se o risco é o responsável pela relação positiva entre taxa de juros e taxa de câmbio. Em outras palavras, uma elevação do risco percebido pelo investidor estrangeiro em títulos brasileiros leva 
a uma depreciação da moeda brasileira, que teria como resultado elevação da inflação, que é combatida com elevação de juros. Dessa forma, ocorreria a relação positiva entre depreciação da moeda e elevação dos juros da política monetária.

Ainda há outras extensões que podem ser feitas a partir desde trabalho para melhor compreender melhor a origem da relação positiva entre taxa de câmbio e taxa de juros, como por exemplo: a inclusão de variáveis dummy para intervenção do Banco Central no mercado de câmbio e a utilização de uma metodologia específica para parâmetros que variam no tempo ou com mudança de regime, a fim de não só verificar o comportamento dos parâmetros em diferentes perfis de risco, como também incluir variáveis que captam os fundamentos da economia e permitem comparações com demais países.

\section{REFERÊNCIAS}

ARAGÓN, E. K. D. S. B.; PORTUGAL, M. S. Central Bank preferences and monetary rules under the inflation targeting regime in Brasil. Brazilian Review of Econometrics, v. 29, n. 1, p. 79-109, 2009.

BACKUS, D.; DRIFFILL, J. Rational expectations and policy credibility following a change in regime. Review of Economic Studies, v. 52, n. 2, p. 211-221, 1985.

BLANCHARD, O. Fiscal dominance and inflation targeting: lessons from Brazil. NBER Working Paper, n. 10389, 2004.

CAPORALE, G.; CIPOLLINI, A.; DEMETRIADES, P. Monetary policy and the exchange rate during the Asian crisis: identification through heteroskedasticity. Journal of International Money and Finance, v. 24, p. 39-53, 2005.

CARVALHO, F. A.; MINELLA, A. Survey forecasts in Brazil: a prismatic assessment of epidemiology, performance, and determinants. Journal of International Money and Finance, v. 31, n. 6, p. 1371-1391, 2012.

COUDERT, V.; GEX, M. Does risk aversion drive financial crises? Testing the predictive power of empirical indicators. Journal of Empirical Finance, v. 15, p. 167-184, 2008.

CURADO, T.; CURADO, M. Identificando as preferências do banco central do Brasil (20022013). Estudos Econômicos, v. 44, n. 3, p. 445-467, 2014.

MENDONÇA, H. F. Mecanismos de transmissão monetária e a determinação da taxa de juros: uma aplicação da regra de Taylor ao caso brasileiro. Economia e Sociedade, v. 16, p. 65-81, 2001.

DEVEREUX, M. B.; ENGEL, C. Monetary policy in the open economy revisited: Price setting and exchange-rate flexibility. The Review of Economic Studies,v. 70, n. 4, p. 765-783, 2003.

DORNBUSCH, R. Expectations and exchange rate dynamics. Journal of Political Economy, v. 84, n. 6, p. 1161-1176, 1976. 
ENGEL, C. Local-currency pricing and the choice of exchange-rate regime. European Economic Review, v. 44, n. 8, p. 1449-1472, 2000.

ESPINOZA, R.; SEGOVIANO, M. Probabilities of default and the market price of risk in a distressed economy. IMF Working Paper, WP/11/75, 2011.

FRENKEL, J. A.; MUSSA, M. L. Asset markets, exchange rate and the balance of payments. NBER Working Paper, n. 1287, 1984.

FURMAN, J.; STIGLITZ, J. Economic crises: evidence and insights from East Asia. Brookings Papers on Economic Activity, p. 1-135, 1998.

GOLDFAJN, I.; GUPTA, P. Does monetary policy stabilize the exchange rate following a currency crisis? IMF WorkingPaper, WP/99/42, 1999.

GONÇALVES, C. E.; GUIMARÃES, B. Monetary policy, default risk and the exchange rate. Revista Brasileira de Economia, v. 65, n. 1, p. 33-45, 2011.

GOULD, D.; KAMIN, S. B. The impact of monetary policy on exchange rates during financial crises. International Finance Working Paper, v. 669, 2000.

HILSCHER, J.; NOSBUSCH, Y. Determinants of sovereign risk: Macroeconomic fundamentals and the pricing of sovereign debt. Review of Finance, v. 14, n. 2, p. 235-262, 2010.

HNATKOVSKA, V.; LAHIRI, A.; VEGH, C. A. The exchange rate response puzzle. University of British Columbia and University of Maryland, 2011. Mimeo.

KOHLSCHEEN, E. The impact of monetary policy on the exchange rate: A high frequency exchange rate puzzle in emerging economies. Journal of International Money and Finance, v. 44, p. 69-96, 2014.

KOURI, P. J. K. The exchange rate and the balance of payments in the short-run and in the long-run: a monetary approach. Scandinavian Journal of Economics, v. 78, n. 2, p. 280-304, 1976.

PALMA, A. A.; PORTUGAL, M. S. Preferences of the Central Bank of Brazil under the inflation targeting regime: Estimation using a DSGE model for a small open economy. Journal of Policy Modeling, v. 36, n. 5, p. 824-839, 2014.

RIGOBON, R. Identification through heteroskedasticity. Review of Economics and Statistics, v. 85 , n. 4, p. 777-792, 2003.

RIGOBON, R.; SACK, B. The impact of monetary policy on asset prices. Journal of Monetary Economics, v. 51, p. 1553-1575, 2004.

STIGLITZ, J. E. Interest rates, risk, and imperfect markets: puzzles and policies. Oxford Review of Economic Policy, v. 15, n. 2, p. 59-76, 1999.

TAYLOR, J. B. The role of the exchange rate in monetary-policy rules. The American Economic Review, v. 91, n. 2, p. 263-267, 2001.

ZETTELMEYER, J. The impact of monetary policy on exchange rates: evidence from three small open economies. IMF WorkingPaper, WP/00/141, 2000.

ZETTELMEYER, J. The impact of monetary policy on exchange rates: Evidence from three small open economies. Journal of Monetary Economics, v. 51, p. 635-652, 2004. 